\title{
Single-Atom Based Coherent Quantum Interference Device Structure
}

\author{
Borislav Naydenov ${ }^{1,3}$, Ivan Rungger ${ }^{2,3}$, Mauro Mantega ${ }^{2,3}$, Stefano Sanvito ${ }^{2,3}$ \\ and John J. Boland $* 1,3$ \\ ${ }^{1}$ School of Chemistry, Trinity College, Dublin 2, Ireland. \\ ${ }^{2}$ School of Physics, Trinity College, Dublin 2, Ireland. \\ ${ }^{3}$ Center for Research on Adaptive Nanostructures and Nanodevices (CRANN), Trinity \\ College, Dublin 2, Ireland.
}

\begin{abstract}
:
We describe the fabrication, operation principles and simulation of a coherent single-atom quantum interference device (QID) structure on $\mathrm{Si}(100)$ controlled by the properties of single atoms. The energy and spatial distribution of the wavefunctions associated with the device are visualized by scanning tunneling spectroscopy (STS) and the amplitude and phase of the evanescent wavefunctions that couple into the quantum well states are directly measured, including the action of an electrostatic gate. Density functional theory simulations were employed to simulate the electronic structure of the device structure, in excellent agreement with the measurements. Simulations of device transmission demonstrate that our coherent single-atom QID can have ON-OFF ratios in excess of $10^{3}$, with potentially minimal power dissipation.
\end{abstract}

Keywords: Quantum confinement, scanning tunneling spectroscopy (STS), Si(100), DFT, Nanodevice.

Semiconductor device technology is undergoing a revolution in device design. Present-day fin-shaped devices will soon give way to 1-d nanowire-like channels in a gate-all-around configuration. The latter, fabricated on silicon-on-oxide (SOI) substrates [1], will enable higher integration densities and enhanced performance. Whereas MOSFET devices are charge based 
involving high levels of power dissipation, the emerging 1-d channel form factors provides opportunities to explore new kinds of device operation. In particular, with channel lengths now approaching $10 \mathrm{~nm}$ the possibility of fabricating coherent quantum interference devices (QID) become feasible, where device operation is based on the phase relationship between the wavefunctions in different regions of the device and not on the charge flowing through it.

Previous attempts to fabricate 1-d QIDs exploited carbon nanotubes as the channel material $[2,3]$. Resonators were defined using barriers formed by unknown defects [4] present in the tubes [5] or by source and drain contacts [6] to the tube itself [7]. These nanotube devices exhibit low ON-OFF ratios and poor reproducibility, the latter caused by an inability to precisely control the coupling to the resonator, which leads to unwanted charging and Coulomb blockade effects [8]. The length of the resonator cavity in these nanotubes devices was typically hundreds of nm, so device operation was limited to cryogenic temperatures. Here we employ as a resonator a surface quantum well which differs from the quantum confinement previously reported on metal [9] and semiconductor [10] surfaces, in that it has semitransparent walls that allow coupling with the surrounding Bloch states.

This letter describes the fabrication, operation principles and simulation of a coherent single-atom-based QID on $\mathrm{Si}(100)$, whose dimensions and operation are controlled by the properties of single atoms, thus ensuring the highest possible device fidelity. The energy and spatial distribution of the wavefunctions associated with the device are visualized by scanning tunneling spectroscopy (STS) and the amplitude and phase of the evanescent wavefunctions that couple into the quasi 1-dimensional surfaces states outside the quantum well are directly measured. Electrostatic gating is demonstrated and directly visualized by STM. Density functional theory (DFT) simulations were employed to simulate the electronic structure of the device, in excellent agreement with the measurements. Simulations of the device transmission coefficient, $T$, demonstrate that quantum well (QW) based coherent single-atom QID devices can potentially have ON-OFF ratio levels in excess of $10^{3}$, with expected minimal power dissipation. 


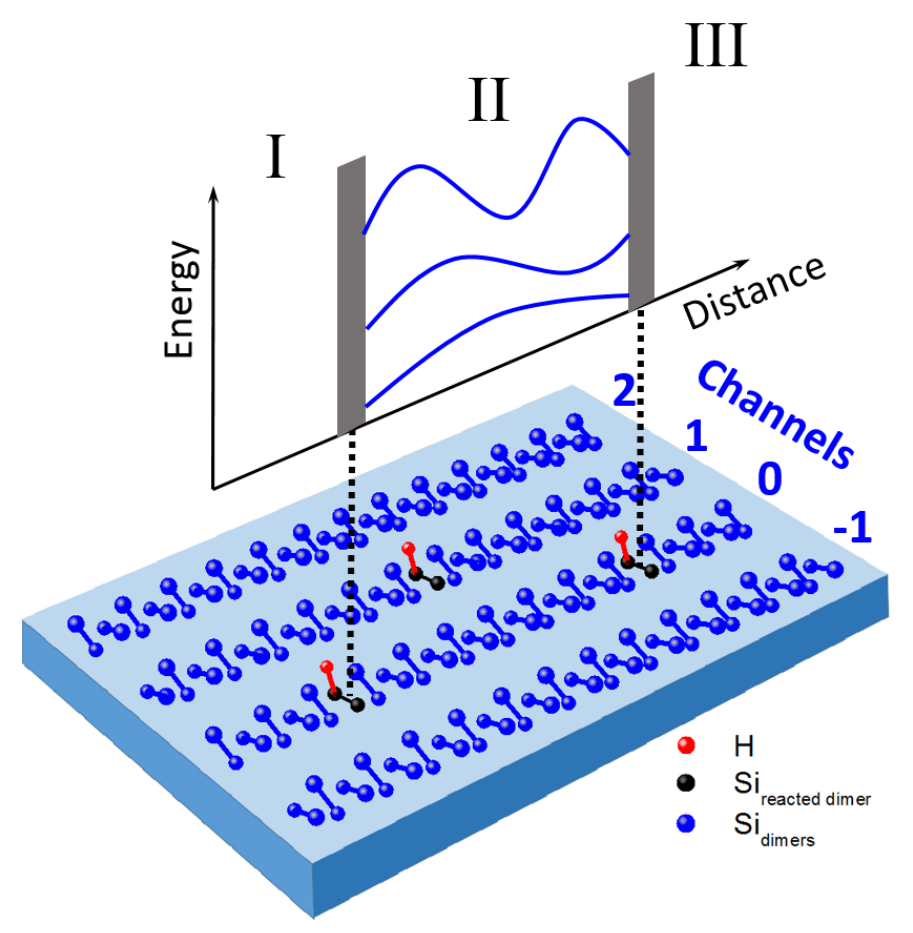

Figure 1. Schematics of the $\mathrm{Si}(100)$ reconstructed surface with the QID and showing the Si dimer rows (blue) together with the QW (channel 0) formed between hydrogen (red) reacted dimers (black). Above channel 0 the wave functions of the QW are plotted.

QID operation involves coherent scattering from two semi-transparent barriers in the device channel (see Fig 1). The barriers are positioned at a separation of 5-10 $\mathrm{nm}$ so as to create a confinement region (II) with well-defined energy levels. Electrons in regions I and III that impinge on the confinement region are scattered and transmitted according to their energy and phase, with the latter determined by the properties of the atomic scattering barriers. The transmission characteristics of the device are thus determined by the precisely defined phase relationship across the three regions that comprise the device.

Rather than engineering a nanowire on SOI [11] and attempt to form a 1-d channel, we exploit the intrinsic anisotropy that exists on $\mathrm{Si}(100)$. The [011] direction is comprised of parallel dimer row channels each characterized by a strongly dispersing electronic state that is largely confined within the Si bulk bandgap. The dispersion in the perpendicular direction is very weak so that these dimer row channels are 1-d in nature, being only weakly coupled to each other and to the underlying bulk Si. 


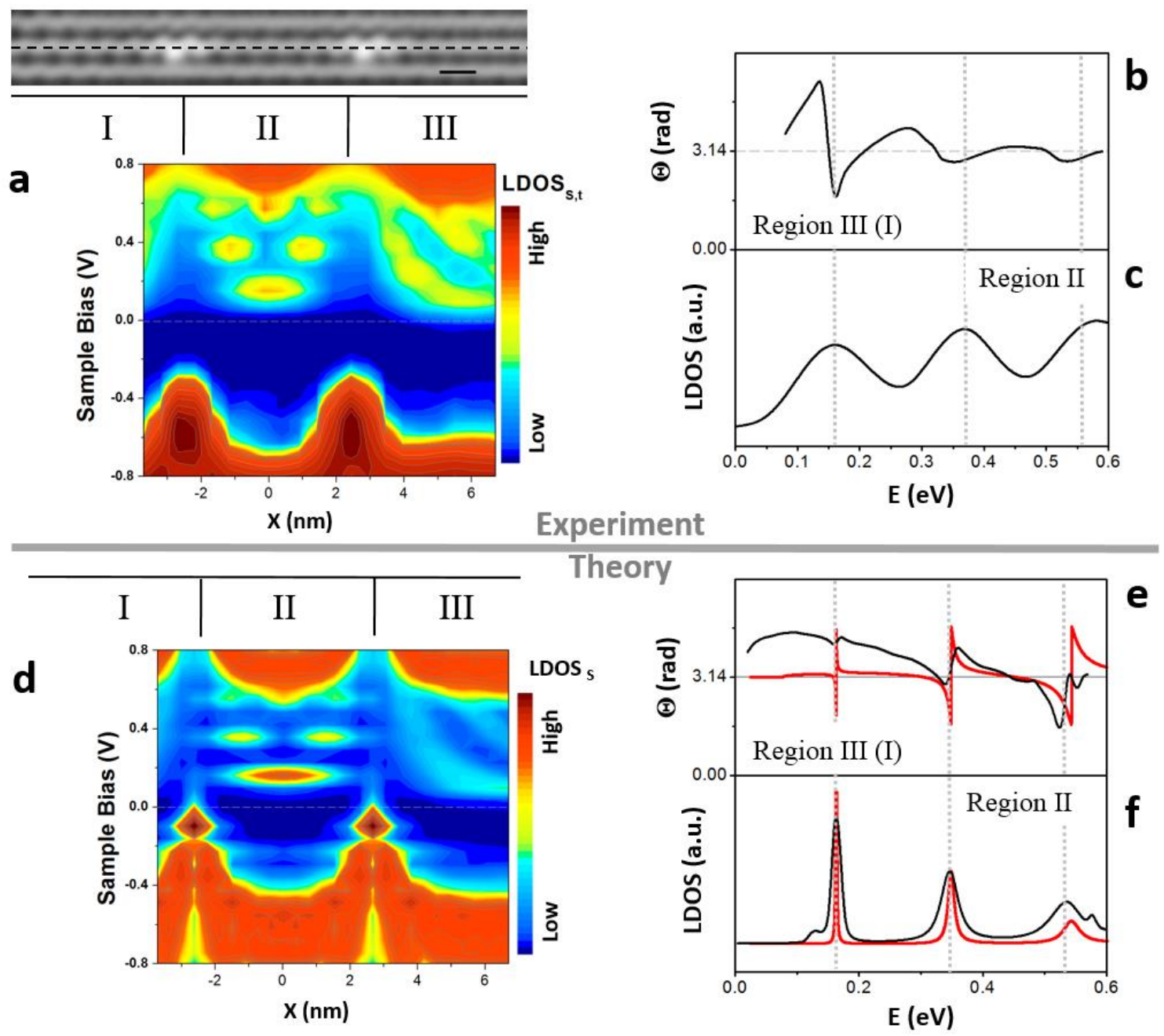

Figure 2. Experimental (a) and calculated (d) LDOS maps of a QW formed by two $\mathrm{H}$ atoms adsorbed on a dimer row. (c) and (f) are the experimental and calculated averaged LDOS spectra across the corresponding QWs. (b) and (e) are the corresponding phase shifts obtained by fitting the standing wave in regions III in (a) and (d) with equation (1). In (e) and (f) the results of the DFT calculations are in black together with red spectra obtained from a simple 1-d model (see text). For better comparison the positions of the energy levels in region II are marked with vertical dotted lines in (b) and (c) and in (e) and (f). The topographic image on the top represents experimental data taken with $35 \mathrm{pA}$ and $-2 \mathrm{~V}$, the scale bar is $1 \mathrm{~nm}$. The spectra in (a) were obtained along the dashed line in the STM image (top-left).

We begin by characterizing the influence of pairs of single-atom scattering centers on the electronic structure of the channel. Fig $2 \mathrm{a}$ and $2 \mathrm{~d}$ show the electronic structure along the channel 
between the two H atoms (see Fig 1) measured by STS and simulated by DFT, respectively. The $\mathrm{H}$ atom reacts with the lower $\mathrm{Si}$ atom of the dimer [12] and creates a single dangling bond (DB) on the adjacent $\mathrm{Si}$ atom of the dimer. $\mathrm{An} \mathrm{n}+$ doped $\mathrm{Si}(100)$ substrate is employed so that the $\mathrm{DBs}$ are doubly occupied and therefore negatively charged [13]. Two filled DB levels are therefore found below the Fermi energy at the location of the dimers containing the $\mathrm{H}$ atoms. Above the Fermi energy the $\mathrm{H}$ atoms lead to well-defined energy levels within a $\mathrm{QW}$, where the $\mathrm{n}=1,2$ and 3 levels are clearly seen (regions II in Fig. 2a and 2d) and the spatial distribution and amplitude of the associated wavefunctions have the expected nodal structure. The uniform energy spacing $(\sim 200$ $\mathrm{meV}$ ) is controlled by the well length and insures that the QID device can in principle operate at room temperature. There is excellent agreement between experiment (Fig. 2a) and simulation (Fig. 2d). Both reveal the presence of structure within the evanescent decay (see region III in Fig. 2a, 2d), particularly at energies associated with the QW states. These structures are related to the barrier height and width associated with individual atoms which allows significant coupling of the QW (II) to the outer regions (I and III), thereby eliminating charging and Coulomb blockade effects. In order to investigate this coupling we have analyzed both experimental and simulated LDOS maps in Fig. $2 \mathrm{a} / 2 \mathrm{~d}$ to determine the phase $\Theta(\mathbf{E})$ and wavevector $\boldsymbol{k}$ of the evanescent decaying standing waves in region III within the $0-0.6 \mathrm{eV}$ energy range using the equation:

$$
\text { LDOS } \propto|\psi(x)|^{2} \propto \text { const }+2 r \cos (2 k x+\Theta)
$$

The results for $\Theta(E)$ determined from experiment and theory are shown in Fig $2 \mathrm{~b}$ and $2 \mathrm{e}$. The experimental and theoretical LDOS of the QW over this same energy range are shown in Fig. 2c and $2 \mathrm{f}$ and comparison of these panels reveal the coupling between the QW states and the evanescent scattering states on either side. This coupling is critical to the device operation. Qualitatively similar phase shifts (red curve in Fig. 2e) are found for a simple 1-d continuum model with a rectangular barrier, with parameters extracted from the DFT transport calculations (see discussion of Fig.5), although the features are sharper since no broadening due to finite temperature and inter-row coupling is included. Significant phase shift variations of the standingwaves are observed whenever the incident energy coincides with one of the QW energy levels. The effect represents the reduced scattering of the QW at its characteristic energy states where less reflection combines with the incidence wave to produce a reduction in the amplitude of the 
scattered standing waves. This resonance scattering accounts for the phase changes seen at incident energies that correspond to QW eigenstates.

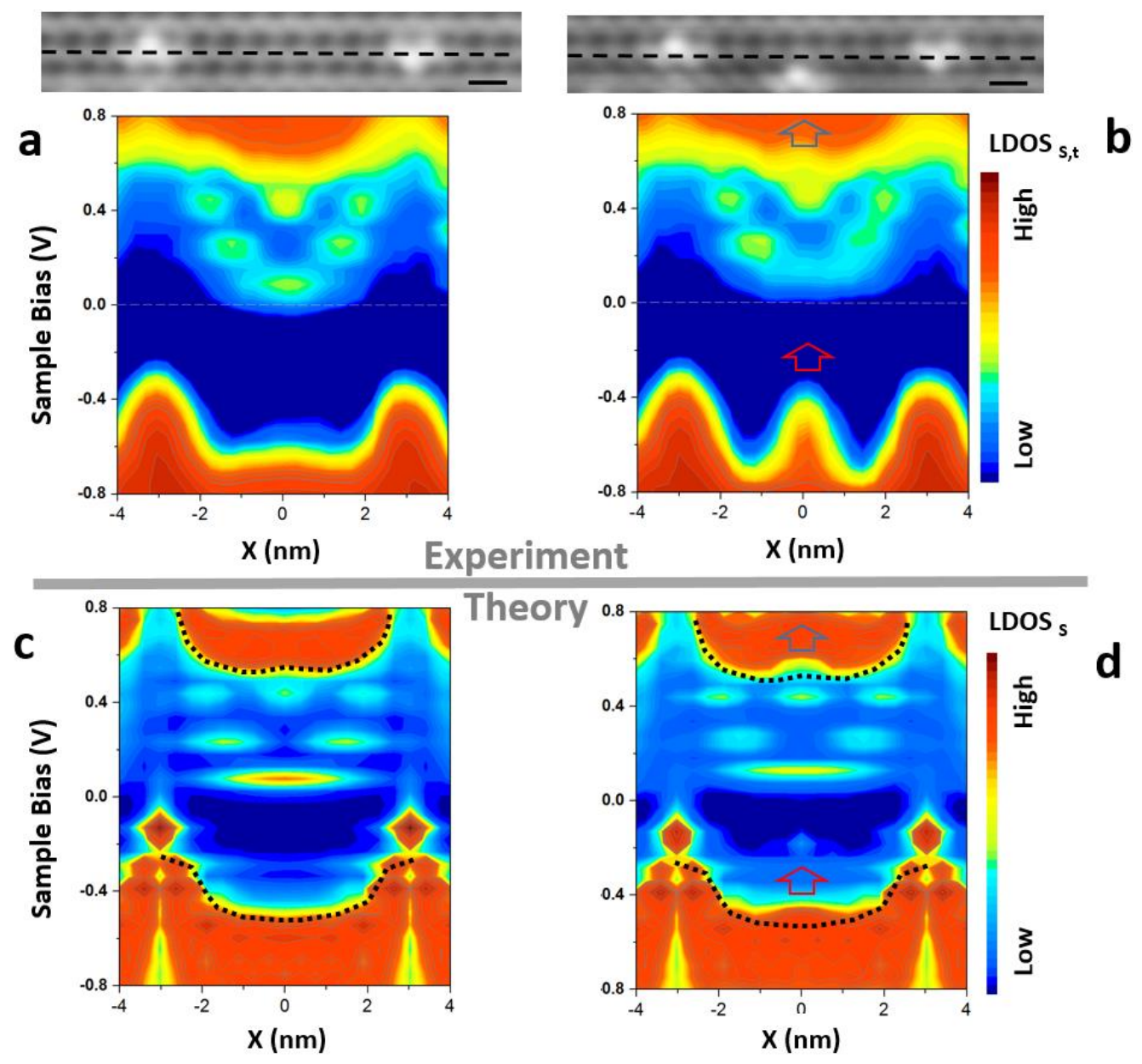

Figure 3. Experimental $(\mathbf{a}, \mathbf{b})$ and calculated $(\mathbf{c}, \mathbf{d})$ LDOS maps for $(\mathbf{a}, \mathbf{c})$ quantum well formed by two $\mathrm{H}$ atoms adsorbed on a dimer row, and $(\mathbf{b}, \mathbf{d})$ same two $\mathrm{H}$ atoms as in (a, c), with a third $\mathrm{H}$ adsorbed on the neighboring row in the middle of the quantum well. The gating action is represented by arrows in (b) and (d). The slight asymmetry in (b) is due to the fact that the absolute middle of the QW is localized between two dimers while the gate is on one of the dimers. The topographic images on the top represent experimental STM data taken with $35 \mathrm{pA}$ and $-2 \mathrm{~V}$ (the scale bar is $1 \mathrm{~nm}$ ). The spectra in (a) and (b) were obtained along the dashed lines in the corresponding STM images (top). Dotted lines in (c) mark the edges of the conducting and valence bands and are repeated in (d) to illustrate better the band bending induced by the gate.

To demonstrate gating of our device channel we used the electrostatic field created by a 
charged DB [14] formed after $\mathrm{H}$ atom adsorption on the neighboring dimer row. The effect is evident from Fig. 3b/3d, where the LDOS along the gated channel and the influence of filled DB state associated with the gating $\mathrm{H}$ atom is seen directly below the Fermi level within the QW, albeit at a lower intensity level that of the DBs that define the QW itself. Experimentally, this was accomplished by using the STM probe to remove and/or reposition an $\mathrm{H}$ atoms, resulting in a $\mathrm{QW}$ with even number of dimers and consequently a slightly asymmetric gating configuration, in contrast with the symmetric configuration of our model. In the Supp. Info. [15] we present additional gating experiments and calculations for a highly asymmetric configuration, and for a significantly longer QW. Although the gating action differs somewhat in the degree of electrostatic localization (see arrows in Fig. 3b and 3d), the gate-induced upward shifts of the QW states is found for both theory and experiment. This can be seen more clearly in Figs. $4 \mathrm{~b}$ and $4 \mathrm{c}$, where the LDOS averaged over the QW is plotted for the non-gated (black) and gated (red) system for the experimental (b) and calculated (c) data in Fig.3. Note that the gating effect depends strongly on the doping level: the higher the doping, the shorter the screening length, and the smaller the gating effect. To illustrate this we performed DFT calculations using various doping levels. Indeed we find a significant dependence of the QW-states position on the doping, as shown in Fig.4a (lines), and where they are also compared with the experimental results from Fig.3a and 3b. Good match between theory and experiment is observed at doping of around $\boldsymbol{n}_{\mathbf{0}}=6.5 \times 10^{19} \mathrm{~cm}^{-3}$ (which is the value used for Fig. 3), both for the gated and non-gated QW. Fig. 4 also shows that the QW peaks are shifted non-uniformly by the gate action, with the lowest energy state undergoing the biggest modification, reflecting the finite effective length of the gate potential (see Fig.3b, 3d, and Ref. [15]). These combined results confirm that the gating effects are tantamount to local band bending induced by the Coulomb potential from charged $\mathrm{DB}$ of the $\mathrm{H}$ atom gate. 

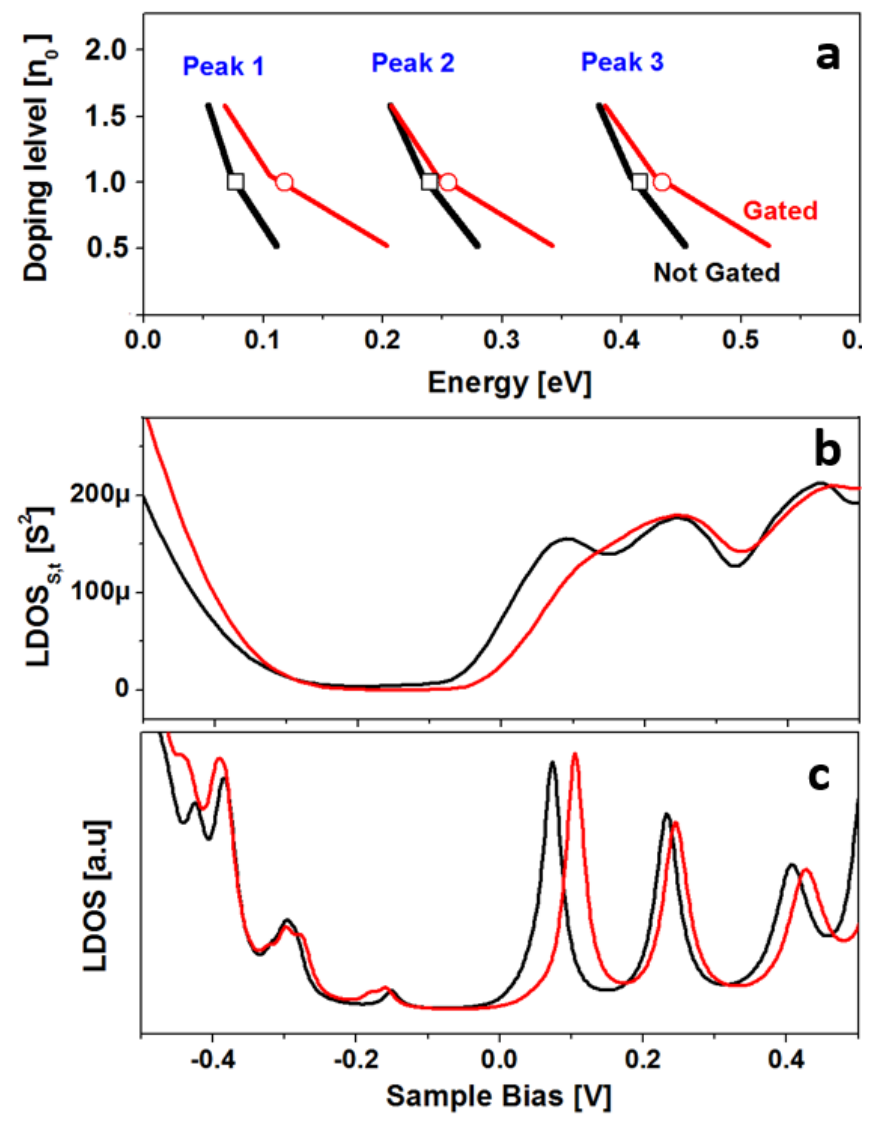

Figure 4. Calculations (a) of the QW states energies for different doping levels (lines) are compared with the experimental peak positions (square for non-gated and circle for gated QW) from Fig.3. The doping level is in units $n_{0}=6.5 \times 10^{19} \mathrm{~cm}^{-3}$. Density of states averaged over the experimental and theoretical QWs presented in Fig.3 (a,b) and Fig.3 (c,d) are plotted in (b) and (c), respectively. Black corresponds to non-gated QW while red marks the gated case in all panels.

In our experimental set-up it is not possible to measure directly the transport properties of the device described above. Instead, we characterize it by using our theoretical model, which has already demonstrated above an excellent agreement with the experimentally observed device features. For better analysis the total transmission $T$ is first split into its transmission eigenchannel (TE) contributions [16]. For a single $\mathrm{H}$ atom adsorbed in row 0 (Fig. 4a; see Fig. 1 for a numbering of the rows) we verified by plotting the wave function associated with each TE, that the TE with the highest $T$ is localized on row 2 , while the lowest $T$ is found for row 0 , as expected. The fact that the eigenchannels are localized on individual rows indicates weak coupling between different rows. We then map the TE transmission for row 0 to a continuum 1-d model with a rectangular 
barrier, using parameters for the barrier height, $V_{\mathrm{B}}$, the barrier length, $l_{\mathrm{B}}$, and the effective mass of the electronic bands inside the barrier region, $m_{\mathrm{B}}$. For the dispersion of the scattering states and corresponding effective mass, $m_{\mathrm{s}}$, we use the DFT band structure of the bare surface. With $V_{\mathrm{B}}=0.49$ $\mathrm{eV}, l_{\mathrm{B}}=1.9 * l_{\mathrm{c}}\left(l_{\mathrm{c}}=7.566 \AA\right.$ ), and $m_{\mathrm{B}}=1.75 m_{\mathrm{s}}$, we obtain a good match to the DFT transmission (red dashed curve in Fig. 4a). Note that we also qualitatively match DFT-NEGF results for the phase shifts across the barriers, as shown in Fig. 2f. Quantitatively they differ, mainly because in Fig. $2 \mathrm{f}$ we add an additional broadening in the DFT-NEGF results, but also due to the inherent limitations of this simple 1-d model when compared to the full DFT-NEGF 3-d potential and scattering.


Figure 5. Eigenchannels transmissions for (a) a single $\mathrm{H}$ adsorbed, (b) two $\mathrm{H}$ atoms adsorbed at a distance $d+l_{\mathrm{B}}$, and (c) two $\mathrm{H}$ atoms adsorbed at a distance $d+l_{\mathrm{B}}$, with a third $\mathrm{H}$ adsorbed on the neighboring row in the middle of the quantum well. The calculated data are for a doping level of $0.5 n_{0}$, which gives the largest gating effect in our calculations; results for the other doping levels are shown in the Supp. Info. [15]. The red dashed lines show the transmission for the corresponding 1-d models with rectangular barriers indicated above the transmission graphs. In (a) where the correspondence between transmissions and rows are well defined the channels are labeled according to Fig. 1. Current versus voltage characteristics of a QW attached to a free-electron-like 1-d metal are shown in (d), and in (e) the resulting bias dependent ON-OFF ratio is presented. 
Using this model we construct the 1-d double barrier potential, and compare the data to the TEs results for the case with two $\mathrm{H}$ atoms adsorbed on row 0 (Fig. 4b). The TE with the lowest $T$ is mainly localized on row 0 , and its transmission matches well with the 1-d model. QW states are formed in row 0 and also in the neighboring rows, especially at low energies, and are visible as peaks in the corresponding TEs. Note that the TEs are not completely localized on individual rows as for the single $\mathrm{H}$ case, which is visible in the avoided crossings in Fig. $4 \mathrm{~b}$. This increased interrow transmission is due to multiple scattering processes that also lead to $\mathrm{QW}$ formation. On row 0 , the lowest energy QW state is only weakly coupled to the electrodes, and has a full width at half maximum (FWHM) of $0.66 \mathrm{meV}$, while the second peak has a FWHM of $6.7 \mathrm{meV}$, and the third one a FWHM of $22 \mathrm{meV}$, reflecting the larger probability for higher energy electrons to be transmitted across the barrier (Fig. 4a).

Adding a single $\mathrm{H}$ atom on the neighboring row 1 results in electrostatic gating, visible as a shift of the peaks to higher energies, while their width is largely preserved (Fig. 4c). Addition of a charge induced gate potential inside the $\mathrm{QW}$ region, $V_{\mathrm{G}}$, of $0.08 \mathrm{eV}$ over a length $l_{\mathrm{G}}=6^{*} l_{\mathrm{c}}$ in the 1-d model reproduces the DFT effective gating. Note that both $V_{\mathrm{G}}$ and $l_{\mathrm{G}}$ become smaller as the doping level is increased (see Supp. Info. [15]), which is due to the better screening. Given that our simulated QW is symmetric, $T$ at the QW state energies is always 1, and to a first approximation the ON-OFF ratio is therefore given by one over the value of $T$ in the minima of the transmission curves, which is $4.0 \times 10^{-4}$ between the first and the second QW state energies, $1.2 \times 10^{-2}$ between the second and the third, and $7.5 \times 10^{-2}$ between the third and the fourth. This gives ON-OFF ratios of 13 to 2500 assuming fully $1-\mathrm{d}$ scattering for the doping level of $0.5 n_{0}$ considered here. A reduction of the doping level is expected to further increase the ON-OFF ratio, while on the other hand including inter-row transmission we expect that these values will be somewhat reduced. Nonetheless this points to QID performance with good ON-OFF ratios and minimal charge dissipation other than that associated with gate charging. To evaluate the current in a possible device setup where the QW is connected to metal wire electrodes, we use our 1-d model, adding free-electron-like band structures outside of the QW barriers. The current shows a step-like increase whenever a QW state enters the bias window, so that the onset of current happens at higher bias for the gated system when compared to the non-gated one (Fig. 5d). The current ON-OFF ratio is bias dependent (Fig. 5e), and reaches a maximum value of about 600 at 0.25 Volt, where 
only the non-gated lowest QW state is inside the bias window.

In summary, we have shown that it is possible to fabricate single atom coherent QIDs, to visualize in real space the phase coupling between the device and the leads and to accurately simulate device LDOS and transmission performance using state-of-the-art DFT under bias. The precise nature of the atom-scale barriers that define the device overcomes the limitations associated with channels defined by uncontrolled defects or the source-drain contacts. The ability to position or pattern atoms or molecules as barriers should allow room temperature device operation and provide an impetus to device community to investigate QIDs as a possible low energy device design.

Methods. Experiments were performed on an n-type Si(100) crystal (As, 0.001-0.005 $\Omega \mathrm{cm})$ at $77 \mathrm{~K}$ using a Createc cryogenic-STM UHV system described elsewhere [16]. Variableheight scanning tunneling spectroscopy [17] was used for all STS measurements. The scattering properties of the single $\mathrm{H}$ atom are obtained with the order- $N$ version of the Smeagol code [18-20], which combines DFT with the nonequilibrium Green's function (NEGF) formalism. We consider the generalized gradient approximation (GGA) to the exchange and correlation functional [21]. The slab used is $9 \mathrm{Si}$ monolayers (plus a passivating hydrogen layer at the bottom) thick, while the unit cell is four dimer rows wide (30.264 $\AA$ ) and extends $204.28 \AA$ along the dimer rows (4752 atoms in total, plus the adsorbed $\mathrm{H}$ atoms). A $20 \AA$ vacuum region is used to avoid interaction between periodic images. We note that for finite thickness slabs the GGA-DFT gap between bulklike states is larger than at infinite thickness [14, 22]. Importantly, the main effect of increasing the slab thickness is a shift of the valence band to higher energies, while the band structure of the surface states, which determines the device properties studied in this manuscript, is largely unaffected. The $n$-type doping conditions are simulated by adding 0.1 electrons for each primitive cell of the $c(4 \times 2)$ reconstructed surface (which contains $72 \mathrm{Si}$ atoms and has a length $l_{\mathrm{c}}=7.566$ $\AA$ ), and a uniform compensating background is added over the slab volume. Details of the basis set and local atomic relaxations around a $\mathrm{H}$ atom are given in Ref. [12]. For the local density of states simulations we add a broadening of $10 \mathrm{meV}$.

\section{Corresponding Author}

*E-mail: jboland@tcd.ie 


\section{Acknowledgements}

JJB and SS acknowledge the Science Foundation Ireland funded Principal Investigator Awards (Grant No. 12/IA/1482, Grant No. 07/IN.1/I945) and CRANN. IR acknowledges financial support from KAUST (ACRAB project). Computational resources have been provided by the HEA IITAC project managed by the Trinity Center for High Performance Computing and by ICHEC.

\section{References}

1. Chen, Z.; Farmer, D.; Xu, S.; Gordon, R.; Avouris, P.; Appenzeller, J. IEEE Elec. Dev. Lett. 2008, 29(2), 183.

2. Venema, L.C.; Wildoer, J.W.G.; Janssen, J.W.; Tans, S.J.; Tuinstra, H.L.J.T.; Kouwenhoven, L.P.; Dekker, C. Science 1999, 283, 52.

3. Meunier, V.; Senet, P.; Lambin, Ph. Phys. Rev. B 1999, 60 (11), 7792.

4. Choi, H.J.; Ihm, J.; Louie, S.G.; Cohen, M.L. Phys. Rev. Lett. 2000, 84 (13), 2917.

5. Kong, J.; Yenilmez, E.; Tombler, T.W.; Kim, W.; Dai, H.; Laughlin, R.B.; Liu, L.; Jayanthi, C.S.; Wu, S.Y. Phys. Rev. Lett. 2001, 87, 106801.

6. Buia, C.; Buldum, A.; Lu, J.P. Phys. Rev. B 2003, 67, 113409.

7. Lemay, S.G; Janssen, J.W.; van den Hout, M.; Mooij, M.; Bronikowski, M.J.; Willis, P.A.; Smalley, R.E.; Kouwenhoven, L.P.; Dekker, C. Nature 2001, 412, 617-620.

8. Liang, W.; Bockrath, M.; Park, H. Annu. Rev. Phys. Chem. 2005, 56, 475.

9. Bürgi, L.; Jeandupeux, O.; Hirstein, A.; Brune, H.; Kern, K. Phys. Rev. Lett. 1998, 81(24), 5370.; Fölsch, S.; Hyldgaard, P.; Koch, R.; Ploog, K.H. Phys. Rev. Lett. 2004, 91, 056803.

10. Yokoyama, T.; Takayanagi, K. Phys. Rev. B 1999, 59(19), 12232.; Sagisaka, K.; Fujita, D. Appl. Phys. Lett. 2006, 88, 203118; Sagisaka, K.; Fujita, D. J. Phys. Conf. Ser. 2008, 100, 052002

11. A. T. Tilke, F. C. Simmel, H. Lorenz, R. H. Blick, and J. P. Kotthaus, Phys. Rev. B 68, 075311 (2003).

12. Naydenov, B.; Mantega, M.; Rungger, I.; Sanvito, S.; Boland, J.J. Phys. Rev. B 2011, 84, 195321. 
13. Haider, M.B.; Pitters, J.L.; DiLabio, G.A.; Livadaru, L.; Mutus, J.Y.; Wolkow, R.A. Phys. Rev. Lett. 2009, 102, 046805.

14. Mantega, M.; Rungger, I.; Naydenov, B.; Boland, J.J.; Sanvito, S. Phys. Rev. B 2012, 86, 035318 .

15. Supporting information

16. Paulsson, M; Brandbyge, M. Phys. Rev. B 2007, 76, 115117.

17. Naydenov, B; Boland, J.J. Phys. Rev. B 2010, 82, 245411.

18. Rocha, A.R.; Garcia-Suarez, V.M.; Bailey, S.W.; Lambert, C.J.; Ferrer, J.; Sanvito, S. Nat. Mater. 2005, 4, 335.

19. Rocha, A.R.; Garcia-Suarez, V.M.; Bailey, S.W.; Lambert, C.J.; Ferrer, J.; Sanvito, S. Phys. Rev. B 2006, 73, 085414.

20. Rungger, I; Sanvito, S. Phys. Rev. B 2008, 78, 035407.

21. Perdew, J.P.; Burke, K.; Ernzerhof, M. Phys. Rev. Lett. 1996, 77, 3865.

22. Radny, M. W.; Smith, P. V.; Reusch, T. C. G.; Warschkow, O.; Marks, N. A.; Wilson, H. F .; Schofield, S. R.; Curson, N. J.; McKenzie, D. R.; Simmons, M. Y. Phys. Rev. B 2007, 76, 155302. 